\title{
Variabilité spatio-temporelle et organisations socioéconomiques paysannes autour du lac Fitri au Tchad
}

\author{
Médard Ndoutorlengar, Université de Sarh, Tchad \\ Pierre Dondonne Goudourm, Université de Ndjamena, Tchad \\ Victor Mignenan, Université de Moundou, Tchad
}

\section{INTRODUCTION}

Les producteurs se contraignent à une forme de mobilité cyclique et des stratégies d'organisation porteuses de nombreuses conséquences socioéconomiques et écologiques importantes.

Pays tropical, le Tchad bénéficie d'un climat sahélien à deux variantes et trois domaines climatiques définis en fonction de la pluviosité. La durée et la pluviosité de chaque variante déterminent les types d'activités socioéconomiques des producteurs ruraux. En fonction de la position géographique, certaines zones sont naturellement favorisées et d'autres non, comparativement aux autres. Les plus favorisées attirent différentes populations pour des raisons liées aux intérêts de chacun (Delcourt, L., 2010). Le degré d'attractivité de ces zones dépend des potentialités qu'elles offrent et de leur position géographique. Les milieux lacustres font partie de ces zones attractives. Le département du Fitri dans la région du Batha au Tchad offre un écosystème à forte multifonctionnalité grâce à la présence de la Fitri qui tire son nom de celui de la localité qui l'abrite.

\section{CADRE ET MÉTHODES}

\subsection{Cadre de la recherche}

Cet article est réalisé grâce aux contributions financières, matérielles et scientifiques du Projet Grands Ecosystème Lacustres Tchad (GELT). C'est un projet franco tchadien financé par la République Française à travers son ambassade au Tchad dont l'objectif principal est de permettre l'organisation d'un vaste programme multidisciplinaire de recherche et de formation sur cinq grands écosystèmes lacustres tchadiens à savoir le lac Tchad, lac Fitri, le Léré, Iro, et les lacs
Dans ces zones, l'agriculture occupe une place prépondérante dans les activités des producteurs ruraux et se pratique sur deux périodes. La saison des pluies est la période de prédilection de l'agriculture. Mais à cause de la faiblesse de ses rendements et des potentialités hydrographiques qu'offre la zone, elle fait place avec la régression et l'étiage des eaux de débordement du lac, à l'agriculture de contre-saison et/ou au maraichage. La pêche et l'élevage constituent également des importantes sources de revenus des habitants du Fitri.

Pour augmenter les revenus, certains producteurs ruraux pratiquent successivement ou simultanément ces trois activités socioprofessionnelles. Mais la gestion concomitante de la pluriactivité impose une mobilisation des ressources matérielles, financières et humaines. Pour ce faire, les producteurs se contraignent à une forme de mobilité cyclique et des stratégies d'organisation porteuses de nombreuses conséquences socioéconomiques et écologiques importantes.

Ounianga. Son objectif spécifique est de comprendre l'évolution climatique dont les aléas concourent à faire des milieux lacustres des zones de concentration transitoire, saisonnière ou permanente, de populations aux modes d'accès aux ressources distinctes (pêche, élevage, agriculture), parfois complémentaires, souvent concurrents (GELT, 2013). Pour des raisons de sécurité autour du lac Tchad où les terroristes «Boko Haram» sévissent régulièrement, la première mission de terrain, prévue pour ce site, est réorientée sur le lac Fitri. 


\section{L'objectif d'étudier les dynamiques des systèmes de production et de la multiactivité au sein des exploitations et de la multifonctionnalité de l'espace qui induisent les transformations du monde rural dans le Fitri.}

La mission du Fitri a regroupé sur le terrain pendant quarante-cinq jours des chercheurs, des enseignants-chercheurs et étudiants issus des deux pays engagés dans le projet. Les Universités et instituts de recherche qui y sont représentés notamment l'Université de Paris I La Sorbonne, l'Institut de Recherche pour le Développement (IRD) du côté de la France et l'Université de Ndjamena, l'Université d'Ati, l'Université d'Abéché, l'Institut de Recherche Agricole pour le Développement (ITRAD) et Institut de Recherche en Elevage pour le Développement (IRED) du côté du Tchad. Nos travaux relèvent du sixième axe de recherche du projet et se donnent l'objectif d'étudier les dynamiques des systèmes de production et de la multiactivité au sein des exploitations et de la multifonctionnalité de l'espace qui induisent les transformations du monde rural dans le Fitri.

\section{RÉSULTATS ET COMMENTAIRES}

La méthode évoquée plus haut nous a permis de comprendre la variabilité dans l'espace et dans le temps ainsi que ses facteurs d'une part et, les mécanismes et types de production de même que leurs impacts socioéconomiques, d'autre part.

\subsection{Fitri, zone variable dans le temps et l'espace}

Du point de vue administratif, le Fitri est l'un des trois départements de la région du Batha. Il est limité à l'Est par le Batha-Ouest, à l'Ouest et au Sud par le Hadjer-Lamis, au Nord par le Kanem (voir figure $\mathrm{n}^{\circ} 1$ ). Il est composé de deux souspréfectures à savoir Yao et Ndjamena Bilala. Le Fitri est géré par des autorités administratives. À côté de cette administration, règne, à partir de Yao, un sultanat d'autorité fortement ancrée dans la tradition. Le Sultan est le Chef suprême dont le pouvoir est répercuté dans les entités administratives par le Khalifat. Sous ce dernier se trouvent

\subsection{Méthodologie}

La méthodologique mobilisée pour cette recherche est de type exploratoire et de nature qualitative. Les méthodes de collecte de données qui nous ont permis de rédiger ce papier sont basées sur les enquêtes, les entretiens semidirigés et les observations directes de terrain. Les enquêtes sont réalisées dans les différents villages insulaires et des terres fermes de la zone grâce aux moyens de locomotion (véhicules et pirogues motorisées) et aux guides et interprètes mis à notre disposition par le projet. Les questionnaires ont été administrés aux trois différents types d'acteurs de production ruraux à savoir les éleveurs, les agriculteurs et les pêcheurs. Ils ont été administrés aux individus pris isolément tandis que les entretiens ont été faits de manière participative (Entretiens en groupe et les entretiens individuels) au cours desquels les paysans sont réunis dans les villages visités étant donné qu'un paysan peut participer successivement à ces trois activités.

\section{La méthodologique mobilisée pour cette recherche est de type exploratoire et de nature qualitative.}

les Kaidala qui sont les représentants directs du Sultan dans les entités administratives. Après les Kaidala, viennent les Chefs de villages appelés Kadjala et les Boulama qui sont les chefs de carré.

De climat sahélien, située plus au sud de la région du Batha, la zone reçoit une pluviométrie annuelle comprise entre 500 et $600 \mathrm{~mm}$. La moyenne pluviométrique des dix dernières années enregistrée à Yao est de 400,46 mm. Les eaux de ruissellement et du fleuve Batha coulent dans le bassin du lac Fitri. Ce dernier reçoit en moyenne 1 milliard de mètres cube par an dont $60 \%$ proviennent des apports du Batha (Lemoalle, 1996). Le reste des apports provient des principaux canaux d'écoulement qui sont, entre autres, les bahrs Melmele, Zerzer, Zilla et Abourda du massif central tchadien (ATPDI, 2010). Ce qui fait de ce lac le deuxième en étendue et en volume après le lac Tchad. Sa 
superficie est de $420 \mathrm{~km}^{2}$ pendant la période des basses eaux avec une moyenne de $800 \mathrm{~km}^{2}$ pendant celle des hautes eaux. Le niveau du lac peut, selon la pluviosité des années, varier de 2 à $4 \mathrm{~m}$ en fin septembre et début octobre. L'échelle de Yao indiquait 3,59 pour une moyenne de 2,68 m les dernières années (BIEP, 1989; Courel, 1994). Il arrive des cas où elle peut doubler ou tripler pendant les grandes crues selon les zones enquêtées.

\section{Figure 1 - Carte de localisation de la zone d'étude}

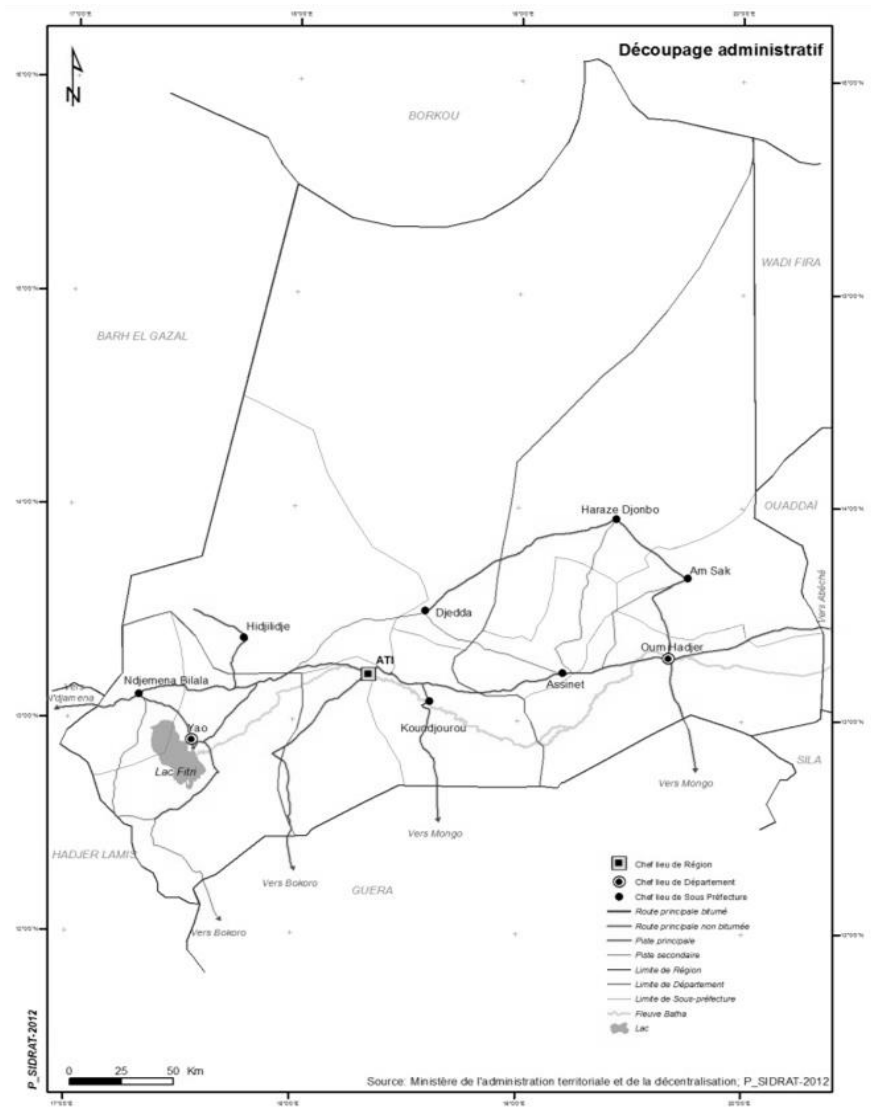

La dynamique des eaux déterminent les types de villages dans la zone du Fitri qui se distinguent en fonction de la taille des ménages, de la durée d'occupation au cours de l'année et de la nature de la principale activité des occupants. On distingue :

\section{Les villages originels ou « villages mères »}

Ils se distinguent des autres par la taille des ménages et le pouvoir traditionnel qui y règne. Le nombre minimum des ménages d'un village originel est de 20. On trouve à l'intérieur de chaque ménage entre 5 et 7 personnes. Le pouvoir traditionnel dans ce type de village est foncièrement ancré. La hiérarchie est respectée et le conflit de compétence n'est pas toléré. Ces villages sont appelés «zone de confiance » pour la raison que quelque soit le type d'inondation due au débordement des eaux du lac, on trouverait dans le village au moins cinq ménages. Ces villages sont dotés de centres de santé et d'écoles stables. Ils bénéficient de quelques aménagements des pouvoirs publics et des projets financés par des bailleurs de fonds.

\section{Les villages insulaires}

Situé entre 280 et $290 \mathrm{~m}$ du niveau de la mère, le Fitri est un véritable amas des îles et des plans d'eaux (ATDP, 2010). Selon l'inspecteur départemental de l'éducation de Yao, le nombre des îles dans la zone varient suivant les saisons climatiques. Pendant la saison des pluies qui favorisent la montée des eaux, on compte 98 îles contre 78 en saison sèche correspondant à la période de retrait des eaux dans les lits majeurs du lac. La 
montée des eaux entraine un débordement qui fait disparaitre 10 îles en saison sèche. De par leur étendue et les activités qui s'y pratiquent, on compte 7 principales îles dans la zone. Il s'agit des Moudo (I et I), Rokawa, Goulou, Wanata, Kessi, Yotto, et Ornio où les activités socioprofessionnelles sont permanentes (voir figure $\mathrm{n}^{\circ} 2$ ). La principale activité sur les îles est la pêche suivie de l'élevage. L'agriculture y est marginale et se résume au maraichage. Ce dernier est favorisé par la présence de l'eau et limité par l'insuffisance de l'espace. La surface des cultures maraichères évolue au rythme de la régression des eaux du lac pendant toute la période de séjour sur les îles.

\section{Figure 2 - Carte altimétrique}

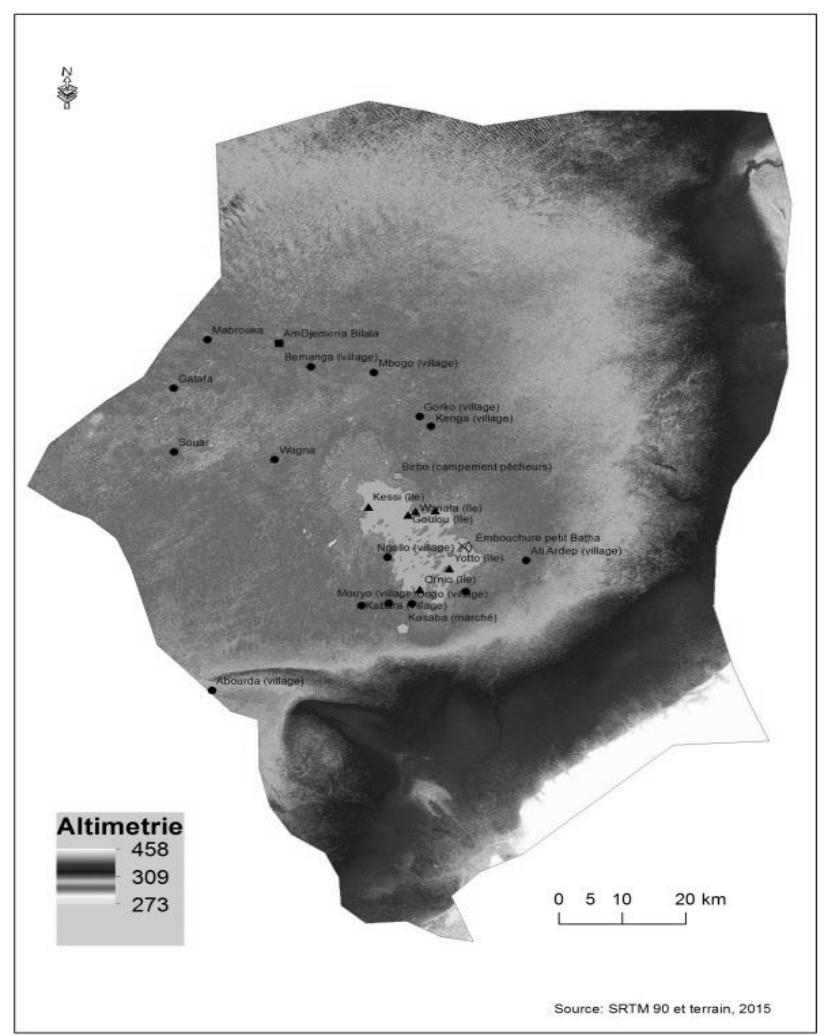

\section{Les villages saisonniers}

Ce sont des villages qui sont aussi primitifs dont la particularité est la mobilité de l'ensemble de ses habitants. Ils se renflouent des populations en saison des pluies et se vident en saison sèche. À l'échelle nationale de la saison des pluies, la durée varie crescendo du Nord vers le Sud. Elle correspond, dans tous les cas, à la période des activités agricoles pluviales. À l'approche de cette période, des exodants pendulaires et les travailleurs saisonniers, dont la principale activité est l'agriculture, se retirent dans leurs villages respectifs. C'est également le cas des ressortissants de la zone du Fitri. À cette période, les habitants des îles qui fuient l'invasion de leur site par la montée des eaux, les éleveurs qui associent l'agriculture à leur activité primaire ainsi que les «boulala » se retirent sur les terres fermes pour labourer. Dans toutes les villes du Tchad, les colporteurs d'eau sont désignés par le mot «boulala» qui est le nom de la principale ethnie du Fitri. En effet, même si d'autres ethnies pratiquent de nos jours cette activité, les ressortissants de celle-ci y restent majoritaires. Les principaux sont, entre autres, Gambir, Darasna, Tassourou, Abourda, Galo, Regnegne (voir figure $n^{\circ} 3$ ).

\section{Les villages satellites ou villages de relais}

Ce sont des camps de passage de court séjour intermédiaire entre les villages insulaires et les villages saisonniers ou encore entre les villages originels et les villages insulaires. Ils sont créés pour 
des besoins de causes qui sont les cultures de contresaison et les maraichères. Ils disparaissent systématiquement à la fin des activités qui rassemblent les habitants sur les lieux. Les habitations sont faites de matériaux précaires. Les populations s'alimentent, pour tous les usages en eau du lac. C'est également le cas des campements des agriculteurs et des pêcheurs. Les ferricks sont les campements des éleveurs qui vivent dans les mêmes conditions, mais se distinguent d'eux par la durée du séjour des habitants sur les lieux.

Chacun de ces villages a un rôle fondamental à jouer dans la vie active des paysans du Fitri. Ils font des mouvements périodiques, chacun en ce qui le concerne, en fonction du type d'activité socioprofessionnelle à laquelle il est attaché.

\section{Figure 3 - Localisation des villes dans le Fitri}

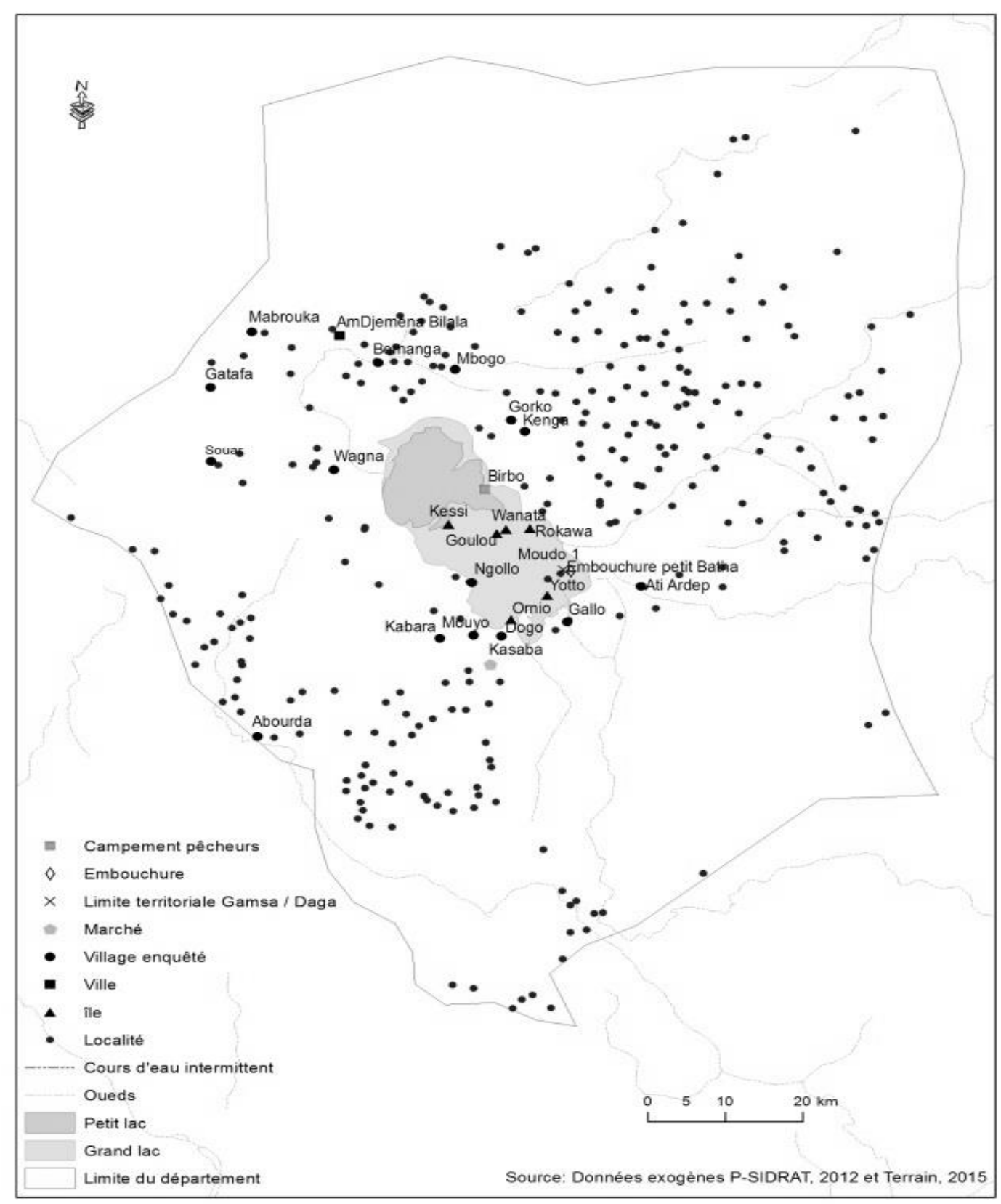




\subsection{Mécanismes de production dans le Fitri}

Les mouvements saisonniers des populations des îles et des villages autour du lac est une caractéristique propre aux paysans de la zone du Fitri. Ils sont cycliques et permanents dans le temps et dans l'espace (voir figure $n^{\circ} 2$ ).

Figure 4 - Cycle de rotation des paysans dans le Fitri

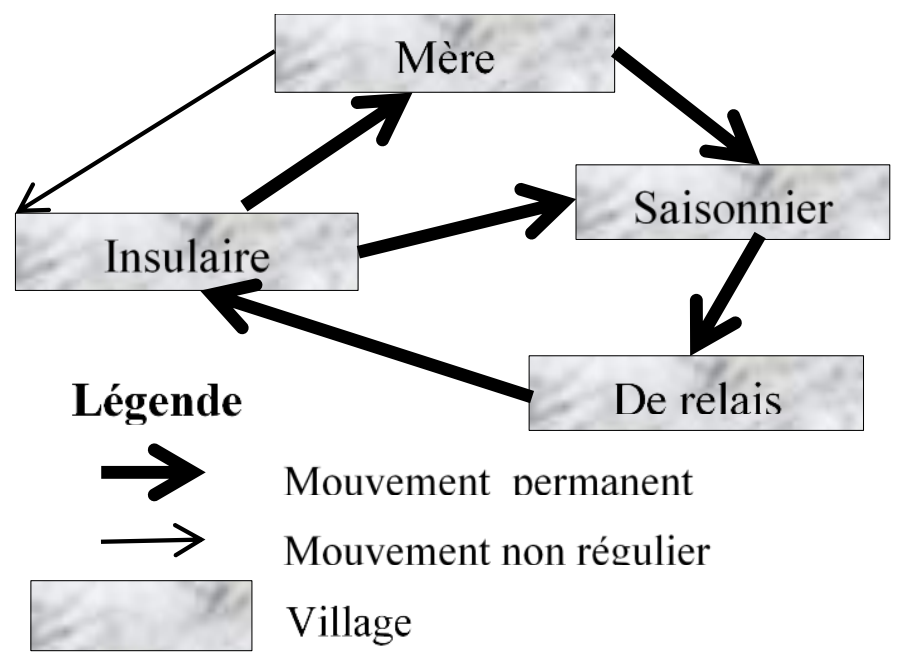

Source : données de terrain, février-mars 2015

Pour expliquer cette figure, nous prenons le départ à partir des villages insulaires. En effet, pendant la saison des pluies, les paysans, toutes les catégories professionnelles confondues, se replient dans les villages soit dans les primitifs soit dans les villages saisonniers. La destination dépend des villages de provenance du paysan. Tous les paysans en activité sur les îles ne sont certainement pas originaires de la région du Batha. C'est l'intensité et la portée bénéfique des activités qui constitue le mobile et l'enjeu de leur arrivée. La date du repli des villages insulaires vers les primitifs ou saisonniers dépend du type d'activité que pratiquent les paysans. Les pêcheurs et les éleveurs qui associent leur principale activité à l'agriculture effectuent leur retrait des îles à partir de la dernière décade du mois de mai à juin. Leur départ précoce est motivé par le besoin de la préparation des champs qui consiste à bruler les anciennes tiges ou couper les épineux qui jonchent le sol. Le domaine est une transition entre le Sahara et le Sahel. Les éleveurs et les pêcheurs de profession quittent un peu plus tard entre juillet et août. Le retrait de cette catégorie de paysans est plutôt pour des raisons sécuritaires aussi bien pour les hommes que pour les troupeaux. À partir du mois de juillet, le lac commence à encaisser les eaux de ruissellement et du fleuve Batha. Avec l'augmentation du niveau d'eau, certaines îles sont submergées et d'autres menacées. La vie y est désormais difficile à cause de l'humidité et de la prolifération des insectes et reptiles. La pêche est également moins productive à cette période des hautes eaux. Les principaux villages de retrait sont Yao, Ndjamena-Bilala, Ambassatna et même audelà de la sous-préfecture voire département où les autorités administratives se retirent elles aussi. En saison des pluies, excepté la famille du Sultan et celles de quelques-uns de ces notables qui restent dans la zone, toute l'administration quitte la zone. C'est en général à Ndjamena-Bilala qu'ils se rendent.

L'agriculture pluviale occupe les paysans dans les villages saisonniers pendant quatre mois, soit de juillet à octobre. Les cultures pluviales les plus dominantes sont les céréales (mil, sorgho, berberé) dont dominent le pénicillaire, le sorgho. À celles-ci s'ajoutent quelques cultures relativement récentes comme l'arachide, le sésame, le niébé et le gombo. La surface cultivée par famille est de 2 à 3 hectares en moyenne. Environ 10000 ha de penicillaire sont emblavés en année normale. Le rendement est de 
$200 \mathrm{~kg} / \mathrm{ha}$ en mauvaise année et de $600 \mathrm{~kg} / \mathrm{ha}$ en bonne année, Le rendement moyen du sorgho est de 600 à 800 ha en année normale (ATPDI, 2010).

Le mois d'octobre marque la fin des pluies dans cette partie sahélienne du Tchad et s'amorce la régression des eaux de divagation du lac. La régression des eaux libère aussi progressivement l'espace propice à la culture de décrue. À cet instant, les paysans quittent les villages saisonniers pour les villages de relais. Les champs dont les cultures ne sont pas arrivées à maturité sont simplement laissés aux femmes et jeunes personnes pour les récoltes. Les lieux et le mode d'implantation des producteurs de décrue dépendent de leurs moyens financiers. Ceux qui ont les moyens s'installent autour des périmètres aménagés par le Programme National de Sécurité Alimentaire (PNSA), un programme présidentiel de visée de développement rural. Il s'agit du périmètre irrigué de Djra de 188 ha de superficie situé à $5 \mathrm{~km}$ au Nord-Est de Yao, celui de Yolo a une superficie de 240 ha placé à $8 \mathrm{~km}$ au Nord-Ouest de Yao et enfin de Touflou (75 ha). Ce dernier se trouve à $3 \mathrm{~km}$ de la même localité. Avec une somme de $10000 \mathrm{f}$ CFA, le paysan peut disposer d'un ha pour la culture de décrue. Ces aménagements ont été réalisés en 2012 par une société chinoise appelée CGCOC. Ces périmètres sont dépréciés par les producteurs déjà après deux années d'exploitation. Selon les paysans, les terres qui ont subi des remuements par les tracteurs sont appauvries. Pour la campagne agricole 2014-2015, les boudeurs des périmètres aménagés et quelques paysans démunis ont simplement choisi d'installer les champs en bordure du lac. Dans les deux cas, les producteurs regagnent les villages de relais à proximité des champs de décrue. L'installation des producteurs aux alentours des champs a double objectif. Le premier est de réduire le temps d'accès aux champs en vue de gagner en quantité et le second est de s'offrir l'avantage d'une meilleure surveillance des champs à leur maturité vis-à-vis des « ennemis des cultures » qui sont les criquets et les oiseaux.

Le système de production du sorgho de décrue ou de béré-béré est différent de celui des céréales pluviales. La culture de béré béré ne demande pas de labour en raison de la surface des terres de culture submergées d'eau pendant plusieurs mois et de surcroit qui contiennent que peu d'adventices au retrait des eaux. Ainsi, le paysan procède simplement au semis sans se préoccuper des défrichages. La plantation peut se faire en semis ou un repiquage après préparation de semis. Le semis se fait quand le sol est encore humide, à l'aide d'un bâton au bout pointu qu'on enfonce dans le sol jusqu'à 25 à $30 \mathrm{~cm}$. Les graines sont ainsi déposées au fond du trou à raison de 4 à 8 graines par poquet. On recouvre ensuite les graines d'une couche de sable. Dans ces conditions, les jeunes pousses sont protégées de certains prédateurs. Les écartements entre les poquets sur une ligne varient de 1,2 à 1,5 mètres (Niangaly Ousmane, 2001). Le repiquage, en ce qui le concerne, est aussi pratiqué au moment du retrait de l'eau comme dans le premier cas après une préparation de la pépinière dont la durée de croissance varie de 1 à 2 mois. Du semis à la récolte, l'entretien du béré béré est moins exigent. Un seul sarclo-binage est exécuté quand les lignes de semis sont bien visibles avant que le sol ne se dessèche complètement. Les paysans ne fertilisent pas les sols pendant la production.

Le maraichage se pratique en suivant les eaux de la décrue à l'étiage. Les principales cultures maraichères rencontrées dans la zone sont la tomate, le gombo, les melons, les pastèques, les courges, les haricots, les concombres et les oignons. Pour ces cultures, les producteurs vont du village de relais aux campements en bordure du lac. Certaines productions maraichères ont été financées par des ONG et des associations de développement rural. Au total 14 groupements féminins ont bénéficié des financements pour la cause. Les principaux appuis financiers au Fitri sont le SECADEV, la Croix Rouge, l'Association Tchadienne pour la Promotion et le Développement Intégré (ATPDI). Il est difficile d'estimer le rendement à cause de l'écoulement progressif des produits mais la taille d'exploitation familiale maraichère est de l'ordre de 0,2 à 0,4 ha. La culture de décrue prend fin avec la récole qui correspond à la période de l'intensification de la pêche. Par contre, le maraichage continue jusqu'à l'aurore des nouvelles inondations. Ce sont souvent les femmes qui continuent la pratique des cultures maraichères pendant ce temps.

Après le maraichage, le mouvement est désormais orienté des villages de relais ou des campements vers les îles pour la pêche où les anciens travailleurs 
de la terre pratiquent la pêche jusqu'à la prochaine montée des eaux qui relance le cycle de la mobilité.

La lutte pour la subsistance et/ou l'amélioration des conditions de vie paysanne dans la zone conduit à une diversification et intensification des activités qui exigent une mobilité permanente des pratiquants desdites activités. Cette façon de faire porte de nombreuses conséquences socioenvironnementales. En tête du peloton des conséquences, se dégage la fracture sociale tant au niveau du noyau familial qu'au niveau de la communauté. En effet, à la maturité des cultures pluviales, les hommes, pour gagner en temps et en espace pour les cultures décrues, migrent vers les villages de relais pour préparer les champs. Les travaux de récolte et de décorticage des produits agricoles sont d'emblées confiés aux femmes et aux jeunes personnes. Il en est de même pour la culture de contre saison où la surveillance, la récolte et les travaux précédents sont assurés par les femmes et les enfants qui, après avoir fini les tâches du village saisonnier, suivent les hommes. L'absence permanente des hommes de leur foyer conduit à une perte d'autorité sur leur progéniture à tous égards. Les enfants, toujours en compagnie des femmes ou seuls dans les champs entrain de surveiller les plantations où paître les troupeaux, se taillent une personnalité que les parents éprouvent souvent de difficulté à reconnaitre. La fracture sociale au niveau communautaire est liée à la problématique du partage des ressources naturelles (FIDA, 2002). Le retour simultané des producteurs ruraux des îles et/ou de la ville augmente le poids démographique sur une terre dont les surfaces agricoles sont tributaires du niveau d'eau. En effet, en année de forte pluviosité, le débordement des eaux du lac envahit certains champs. Entre agriculteurs, il en découle des problèmes de redimensionnement des champs d'une part et, d'autre part, entre agriculteurs et éleveurs les conflits sont liés au confinement des deux catégories professionnelles sur un même espace mettant les troupeaux à proximité des champs dans lesquels ils peuvent faire irruption par inadvertance ou non (Souapibé P. S et al, 2007).

La sempiternelle mobilité qui ne fixe pas les paysans à leurs terroirs respectifs a entrainé un comportement d'indifférence et d'irresponsabilité vis-à-vis de la sensibilité des ressources naturelles. Seule la maximisation du profit est à l'ordre du jour.
Chacune des couches socioprofessionnelles entame les ressources naturelles mais celles dont l'environnement écologique porte les marques visibles sont les éleveurs et les pêcheurs.

En l'absence des herbes, les éleveurs paissent les animaux des feuilles d'arbres. Ce nouveau système de pâturage consiste à élaguer les branches des arbres et/ou couper au niveau du tronc les arbustes autours desquels les animaux sont parqués. Cette pratique prend non seulement de l'ampleur, mais elle ne rencontre pas d'opposition en raison d'une part de l'indifférence et d'autre part des conséquences d'une éventuelle réaction de la part des résidents. Les éleveurs sont, pour la plupart, armés de fusils. Certains troupeaux sont la propriété des auto-rités administratives et militaires qui arment les bouviers d'instruments de dissuasion et de défense.

Également, les ressources halieutiques ne sont pas exemptes des effets de l'intensification, de la mobilité et de la pluriactivité de la zone. En effet, à la période de pointe de la pêche dans le Fitri, le nombre de pêcheurs sur le lac est de 2500 à 3000 dont plus de 600 allogènes (PDL, 2010). En raison de la menace de raréfaction et/ou d'extinction de certaines espèces, depuis près d'une décennie, les autres techniques de pêche ont été interdites par le Sultan au profit de la traditionnelle. La technique traditionnelle de pêche se fait à la palangre constituée d'une ligne formée d'une corde le long de laquelle sont attachés 1000 à 2000 hameçons sans appâts. Les lignes sont posées et relevées au moyen des pirogues en bois de faidherbia albida dans la majorité des cas. Elles sont repérables par des flotteurs d'Aschynomeme elaphroxylon. Les poissons pêchés sont transportés aux bords du lac pour être vendus aux commerçants venus des grandes villes du Tchad ou des pays voisins ou encore sur les îles pour conservation. Il y a deux techniques de conservation des poissons qui sont le séchage et le fumage. Sur les îles, les travaux de conservation sont assurés par les femmes et les enfants pour permettre aux pêcheurs de gagner du temps (Dagou et al., 2005).

\section{Également, les ressources halieutiques ne sont pas exemptes des effets de l'intensification, de la mobilité et de la pluriactivité de la zone.}




\subsection{La mobilité paysanne et la question de l'éducation}

La mobilité permanente des populations de la zone du Fitri a conduit les autorités en charge de l'éducation et les partenaires au développement à repenser le système éducatif et l'adapter au rythme des mouvements des populations. Les écoles sont montées et les ressources financières, matérielles et humaines sont mobilisées pour suivre les paysans dans les différents villages que nous avons énumérés plus haut.

On note deux systèmes d'organisation scolaire dans la zone. Un premier est dédié aux paysans agriculteurs ou agropasteurs et pêcheurs et un autre propre aux éleveurs transhumants. C'est dans les villages originels où sont basées les écoles dument construites et où les enseignants sont régulièrement affectés. C'est également dans ces villages que les écoliers sont régulièrement inscrits. Au niveau national, la rentrée scolaire est, de coutume fixée à la première semaine du mois d'octobre de chaque année. Mais pour de nombreuses raisons, les cours commencent dans la zone au mois de novembre. Chaque année, après un mois de cours, les classes se vident progressivement des apprenants. La raison de cette désertion est que les enfants suivent les parents dans les villages saisonniers.

Les efforts du Programme Alimentaire Mondiale (PAM) qui met régulièrement des vivres pour les cantines pour retenir les apprenants à l'école ont été vains car les raisons de désertion des enfants ne sont pas seulement liées à la faim. Certains sont retirés de l'école par les parents pour servir de main-d'œuvre dans les champs tandis que d'autres quittent les villages originels pour des raisons de sécurité en l'absence des parents.

Pour pallier ces problèmes, les autorités locales en charge de l'éducation ont repensé et mis sur pieds un autre cadre pour l'éducation des enfants. Des écoles sont montées dans les villages saisonniers et de relais appelées «New feet» pour marquer leur caractère transitoire. Ainsi, à chaque élève désireux de quitter l'école du village originel est délivrée par le Directeur une attestation de niveau qui sera présentée à l'école d'accueil. Ces types d'écoles sont tenues pars des enseignants communautaires qui sont des volontaires bénévoles recrutés sur place. Ils sont constitués d'anciens militaires démobilisés, d'anciens élèves n'ayant pas eu la chance de progresser dans les études et dont les niveaux ne sont pas attestés. Certaines de ces écoles sont construites avec des matériaux précaires. D'autres tiennent leurs classes sous les arbres en raison de leur caractère périodique et de leur instabilité. On y rencontre souvent plusieurs niveaux regroupés dans une classe appelée classe polygrade ou un seul enseignant a plusieurs niveaux. Un mécanisme identique est mis en place également sur les îles pour corriger le problème de l'éducation des enfants qui suivent les pêcheurs.

Pour le cas des éleveurs transhumants, des enseignants dits nomades sont affectés à des tribus d'éleveurs appelés «Kachim bet » qui ne sont autre qu'un regroupement de ménage de même origine ethnique. Dans le système des écoles nomades les enseignants suivent les apprenants dans leur déplacement jusqu'à la limite de la circonscription où ils sont affectés. Au-delà de cette limite, le relai est assuré par ceux de la circonscription suivante. Cette pratique du système éducatif porte de nombreuses conséquences dont principalement (Ndoutorlengar Médard, 2014) :

- Le refus d'accepter d'être affecté dans la zone par les enseignants formés fonctionnaire de l'État.

- Le non-respect des programmes et du calendrier de l'année scolaire lié à la multiplicité des acteurs de formation. Dans les villages saisonniers et de relais, l'enseignement appelé la remise à niveau n'est autre que l'apprentissage au calcul et à la lecture.

La baisse de niveau dont l'origine vient de la qualité des enseignants, d'une part et, d'autre part, du non suivi des apprenants par les parents. 


\section{CONCLUSION}

\section{Au chapitre des empreintes sociales du mode d'organisation local, on relève, entre autres, l'effritement du système \\ d'éducation et les conflits sur le partage des ressources naturelles.}

La recherche de l'amélioration des conditions de vie d'une population donnée est une étape vers le développement socioéconomique. Elle en appelle au développement des stratégies de lutte pour l'autosubsistance ou l'augmentation des revenus qui passe de l'intensification à la diversification des activités. Tel est le cas des paysans de la zone du Fitri qui, au-delà des difficultés majeures liées aux phénomènes climatiques communs aux ruraux des pays du Sahel, sont appelés à affronter le temps et l'espace pour survivre.

$\mathrm{Au}$ chapitre des empreintes sociales du mode d'organisation local, on relève, entre autres, l'effritement du système d'éducation et les conflits sur le partage des ressources naturelles. L'empreinte écologique s'exprime par la détérioration du couvert végétal et des ressources halieutiques.

Au rythme actuel d'évolution de la situation, des interventions multisectorielles semblent impératives où l'État, les partenaires au développement ainsi que les populations concernées ont chacun un rôle à jouer. Elles doivent tenir compte des opportunités et des contraintes locales pour réussir.

\section{BIBLIOGRAPHIE ET NOTES}

${ }^{1}$ Delcourt L., (2010). L'avenir des agricultures paysannes face aux nouvelles pressions sur la terre ; Centre Tricontinental (CETRI), article 1806, p. xx-yy. ${ }^{2}$ GELT (2013). Appel à propositions de recherche (2013). Direction de la Recherche Scientifique et Technique, MESRS. 6 pages

${ }^{3}$ Lemoalle. J, (2000). Schéma directeur de l'eau et l'assainissement, secteur hydrologique et environnement aquatique, projet PNUD CHD 98/004, rapport provisoire.

${ }^{4}$ Bureau Interministériel d'Etude et de Programmation (BIEP) (1989): Etude de développement Intégré du lac Fitri. Ministère de l'agriculture, $23 \mathrm{p}$.
${ }^{5}$ Courel M. F., Morin S., Raimond C., (1997). «Intégration modèle ou modèle d'intégration? La gestion de l'environnement au lac Fitri (Tchad) ». In Singaravelou, éd. : Pratiques de gestion de l'environnement dans les pays tropicaux. $16 \mathrm{p}$.

${ }^{6}$ Talence, Dymset-Cret, coll. SD Espaces tropicaux 15 : p. 311-327

${ }^{7}$ Association Tchadien pour la Promotion et le Développement Intégré (ATPDI) (2010). Plan de développement local zone de confiance de Yao souspréfecture de Yao département du Fitri région du Batha, $35 \mathrm{p}$.

${ }^{8}$ Niangaly Ousmane (2001). La culture du sorgho de décrue dans le Nord du Mali, in La culture du sorgho de décrue en Afrique de l'Ouest et du Centre. In La culture du sorgho de décrue en Afrique de l'Ouest et du Centre: In «Situation actuelle et définition d'un Plan d'Action Régional ». p. 143-148.

${ }^{9}$ Chantereau (2001). Connaissance et utilisation de la diversité des sorghos de décrue en Afrique de l'Ouest et du Centre au CIRAD. In "Situation actuelle et définition d'un Plan d'Action Régional ». p. 39-48

${ }^{10}$ Fonds International de Développement Agricole (FIDA) (2002). La gestion de l'environnement et des ressources naturelles. Une préoccupation croissante du FIDA, $48 \mathrm{p}$.

${ }^{11}$ Souapibé Pabamé Sougnabe (2007). Confits agriculteurs-_éleveurs en zone soudanienne au Tchad : une étude comparée de deux régions : Moyen-Chari et Mayo-Kebbi. Actes du colloque, 27-31 mai 2002, Garoua, Cameroun. Mars, 2007. Cirad - Prasac, 8 p. ${ }^{12} \mathrm{P}$. Dagou et al. (2005). La pêche dans les lacs Fitri et Léré au Tchad. Techniques de capture, conservation des produits et enjeux de protection. Ressources vivrières et choix alimentaires dans le bassin du lac Tchad $26: 87-112$. 